\title{
A NEW APPROACH TO MULTIPLE FAULT DIAGNOSIS: A COMBINATION OF DIAGNOSTIC MATRICES, GRAPHS, ALGEBRAIC AND RULE-BASED MODELS. THE CASE OF TWO-LAYER MODELS
}

\author{
Antoni LIGĘZA*, JAN MACIEJ KOŚCIELNY** \\ * Institute of Automatics \\ AGH University of Science and Technology, al. Mickiewicza 30, 30-059 Cracow, Poland \\ e-mail: ligeza@agh.edu.pl \\ ** Institute of Automatic Control and Robotics \\ Warsaw University of Technology, ul. Św. Andrzeja Boboli 8, 02-525 Warsaw, Poland \\ e-mail jmk@mchtr.pw.edu.pl
}

\begin{abstract}
The diagnosis of multiple faults is significantly more difficult than singular fault diagnosis. However, in realistic industrial systems the possibility of simultaneous occurrence of multiple faults must be taken into account. This paper investigates some of the limitations of the diagnostic model based on the simple binary diagnostic matrix in the case of multiple faults. Several possible interpretations of the diagnostic matrix with rule-based systems are provided and analyzed. A proposal of an extension of the basic, single-level model based on diagnostic matrices to a two-level one, founded on causal analysis and incorporating an OR and an AND matrix is put forward. An approach to the diagnosis of multiple faults based on inconsistency analysis is outlined, and a refinement procedure using a qualitative model of dependencies among system variables is sketched out.
\end{abstract}

Keywords: automated diagnosis, diagnostic matrix, diagnostic rules, model-based diagnosis, consistency-based diagnosis.

\section{Introduction}

The diagnosis of multiple faults turns out to be more complex than that of singular faults diagnosis. This is not only because the number of faults increases but also due to the occurrence of new phenomena that must be considered, such as a combination of mutual influence of faults, compensation, and combinatorial explosion of possible failure scenarios. The number of possible diagnoses increases in a significant way since multiple fault diagnoses are elements of the power set of single faults.

Numerous works are devoted to the analysis of methods for singular and multiple fault diagnostic approaches. Some of the popular models include extended diagnostic matrices (Kościelny, 2001; Kościelny, 2004b; Kościelny, 2004a), set-covering model (Reggia, Nau and Wang, 1983; Reggia, Nau and Wang, 1985), consistency-based reasoning (Reiter, 1987; Hamscher, Console and de Kleer, 1992; Ligęza, 2004), logical causal graphs (Fuster-Parra, 1996; Ligęza and Fuster-Parra, 1997; Ligęza, 2004), and many other (Tzafestas, 1989a; Tzafestas, 1989b; Davis and Hamscher, 1992; Korbicz, Kościelny, Kowalczuk and Cholewa, 2004). In the area of Fault Detection and Isolation (FDI), emerging from classical automatic control, these problems are discussed in (Frank, 1990; Frank, 1996; Korbicz et al., 2004).

A recent comparative analysis of the approaches coming from the automatic control area and the FDI community compared with the ones based on Artificial Intelligence (AI) techniques and developed by the DX Community 1 is provided in (Cordier et al., 2000b; Cordier et al., 2000a). It turns out that both of the approaches, developed almost independently by the FDI and DX researchers, are highly parallel, and there are certain far going analogies between analytical redundancy analysis and consistency-based reasoning. Moreover, both of the approaches are based on similar assumptions and use only a model of the correct system behavior. Neither expert nor statistical knowledge or evidence of the fault history is taken into account.

\footnotetext{
${ }^{1} \mathrm{DX}$ is a series of conferences devoted to automated diagnosis methods emerging from AI.
} 
In this paper an analysis of the binary diagnostic matrix approach (Kościelny, 2001; Kościelny, 2004b; Kościelny, 2004a) and its limitations in the case of more complex systems, as well as the occurrence of multiple faults, are briefly discussed. It is argued that some limitations of this model, based on direct, forward interpretation, can be overcome if backward search procedures are applied. Further, the model should be extended in order to cover the case of multiple faults. As a consequence, a new diagnostic model, composed of two types of matrices forming two levels, is developed and presented in detail. The paper builds on ideas initially presented at some conferences, namely, (Ligęza and Kościelny, 2007b; Ligęza and Kościelny, 2007a; Ligęza and Kościelny, 2007c).

One of the matrices defines the so-called intermediate conceptual faults and it is analogous to the classical OR matrix, while the second level matrix combines the influence of the conceptual faults onto manifestations and is a new type AND matrix. The proposed model covers models founded on analytical redundancy and consistency-based reasoning (Cordier et al., 2000b; Cordier et al., 2000a). Simultaneously, it presents a generalization of the classical diagnostic matrix approach (Kościelny, 2001; Kościelny, 2004b; Kościelny, 2004a). A diagnostic algorithm for generating possible minimal diagnoses is also presented here. Moreover, an extension based on the analysis of qualitative influences among the system variables for eliminating certain irrelevant diagnoses is put forward.

Taking into consideration the logical point of view, the presented approach is based on the classical Reiter theory (Reiter, 1987), and incorporates the ideas of AND/OR causal graphs (Ligęza and Fuster-Parra, 1997). It is aimed at an extension and improvement of the FDI approach based on a single OR type diagnostic matrix, which is insufficient in the case of multiple faults diagnosis.

\section{Diagnostic matrices and their logical models}

Traditional binary diagnostic matrices (Kościelny, 2001; Kościelny, 2004b; Kościelny, 2004a) are limited in practice to the diagnosis of single faults. This follows from their internal structure and the assumed operational mode-any such matrix constitutes a set of $K$ patterns (the columns forming the so-called fault signatures) of precompiled knowledge for fast decision making.

Consider a binary diagnostic table of the generic form shown in Table 1 Faults are headers of the columns of the table, while manifestations are labels of its rows.

Consider the $k$-th column of the matrix. Let $g_{i_{1} k}, g_{i_{2} k}, \ldots, g_{i_{k} k}$ be all the elements of this column taking value 1 and hence forming the signature for fault $f_{k}$. The knowledge specified with Table 1 is normally interpreted as a set of forward-chaining rules, each of them of
Table 1 . General scheme of a binary diagnostic matrix.

\begin{tabular}{|c||c|c|c|c|c|c|}
\hline$M / \mathbf{F}$ & $f_{1}$ & $f_{2}$ & $\ldots$ & $f_{k}$ & $\ldots$ & $f_{K}$ \\
\hline \hline$m_{1}$ & $g_{11}$ & $g_{12}$ & $\ldots$ & $g_{1 k}$ & $\ldots$ & $g_{1 K}$ \\
\hline$m_{2}$ & $g_{21}$ & $g_{22}$ & $\ldots$ & $g_{2 k}$ & $\ldots$ & $g_{2 K}$ \\
\hline$\vdots$ & $\vdots$ & $\vdots$ & $\ldots$ & $\vdots$ & $\ldots$ & $\vdots$ \\
\hline$m_{i}$ & $g_{i 1}$ & $g_{i 2}$ & $\ldots$ & $g_{i k}$ & $\ldots$ & $g_{i K}$ \\
\hline$\vdots$ & $\vdots$ & $\vdots$ & $\ldots$ & $\vdots$ & $\ldots$ & $\vdots$ \\
\hline$m_{J}$ & $g_{J 1}$ & $g_{J 2}$ & $\ldots$ & $g_{J k}$ & $\ldots$ & $g_{J K}$ \\
\hline
\end{tabular}

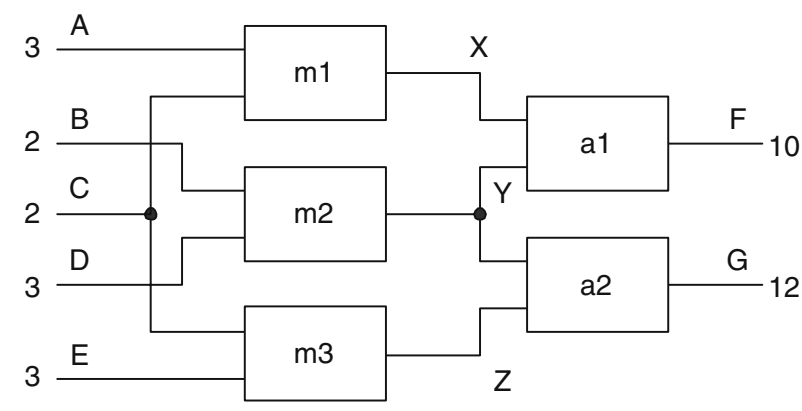

Fig. 1. Exemplary arithmetic system.

the form

$$
\text { rule }_{k}: m_{i_{1}} \wedge m_{i_{2}} \wedge \ldots \wedge m_{i_{k}} \longrightarrow f_{k} .
$$

So, in fact, we have $K$ simple expert-like diagnostic rules interpreted in a single step of inference in the forward direction. However, it turns out that this is not the only possible interpretation. Below, with the example of a multiplier-adder system, several other possible interpretations are provided.

\section{Multiplier-adder example}

Consider the diagnosis of the classical, non-trivial benchmark system being a multiplier-adder system presented in (Reiter, 1987) and further explored in the domain literature (Hamscher et al., 1992; Ligęza, 2004). It is also used for the illustration of the FDI and DX procedures in (Cordier et al., 2000b; Cordier et al., 2000a). The system is presented in Fig. 1. It is composed of two layers. The first one contains three multipliers, $m 1, m 2$, and $m 3$, receiving input signals. The second layer is composed of two adders, namely, $a 1$ and $a 2$, producing the output values. Only the inputs (of the first layer) and outputs of the system (of the second layer) are directly observable. The intermediate variables are hidden and cannot be measured.

In the following, we shall refer mostly to the classical diagnostic problem as follows: The current state of the system is that the inputs are $A=3, B=2, C=2, D=3$ and $E=3$. It is easy to check that-if the system works correctly-the outputs should be $F=12$ and $G=12$. 
Since the current value of $F$ is incorrect, namely, $F=10$, the system is faulty. At least one of its components must be faulty.

3.1. Multiplier-adder: The case of binary diagnostic matrix. Let us analyze the binary diagnostic matrix of the example system. The set of faults will be denoted by $\mathbf{F}$ and the set of manifestations by $M$. There are five potential faults corresponding to failures of the five components. For simplicity, we write $\mathbf{F}=$ $\{m 1, m 2, m 3, a 1, a 2\}$. Further, there are only two diagnostic signals or rather manifestations; for simplicity we denote them as $M=\{F, G\}$.

When considering the signal flow inside the system, it can be observed that the components $\{m 1, m 2, a 1\}$ are the only ones influencing the signal $F$. Similarly, the components $\{m 2, m 3, a 2\}$ are the only ones influencing the signal $G$. The binary diagnostic matrix obtained from such a straightforward causal analysis has the form shown in Table 2, The use of the classical FDI approach based

Table 2. Simple binary diagnostic matrix for the multiplieradder system.

\begin{tabular}{|c||c|c|c|c|c|}
\hline$M / \mathbf{F}$ & $m 1$ & $m 2$ & $m 3$ & $a 1$ & $a 2$ \\
\hline \hline$F$ & 1 & 1 & & 1 & \\
\hline$G$ & & 1 & 1 & & 1 \\
\hline
\end{tabular}

on fault signatures (Kościelny, 2001) and some possibilities of an expert-like and causal interpretation of Table 2 are discussed in (Ligęza and Kościelny, 2007c; Ligęza and Kościelny, 2007b). In this paper we shall follow a more complete model.

3.2. More complete model. A further analysis of the system (Cordier et al., 2000b; Cordier et al., 2000a) allows us to extend the simple matrix through introducing a third residual corresponding to the difference of signals $F$ and $G$. In fact, under the assumed manifestations, one of the elements $\{m 1, a 1, a 2, m 3\}$ must also be faulty. This is so since if all of them were correct, then $Z=C \cdot E$ calculated by $m 3$ must be equal to 6 , and since $G$ is observed to be $12, Y$ (calculated backwards and under the assumption that $a 2$ is O.K.) must also equal 6 ; hence, if $m 1$ is correct, then $X$ must be 6 as well, and if $a 1$ is correct, $F$ would be equal to 12 . Since this is not the case, at least one of the components used must be faulty.

The complete binary diagnostic matrix is given in Table 3. Below we examine the possible rule-based interpretations of the diagnostic matrix given by Table 3 according to the principles discussed in (Ligęza and Kościelny, 2007c; Ligęza and Kościelny, 2007b). The following results are obtained in terms of forward, expert-like and backward, causal type inference rules.
Table 3. Complete binary diagnostic matrix for the multiplieradder system.

\begin{tabular}{|c||c|c|c|c|c|}
\hline$M / \mathbf{F}$ & $m 1$ & $m 2$ & $m 3$ & $a 1$ & $a 2$ \\
\hline \hline$F$ & 1 & 1 & & 1 & \\
\hline$G$ & & 1 & 1 & & 1 \\
\hline$F-G$ & 1 & & 1 & 1 & 1 \\
\hline
\end{tabular}

3.3. Case of forward, expert-like vertical strong diagnostic rules (fevs). Through a simple analysis of the matrix of Table 3, we obtain the following set of strong (i.e., taking into account both the occurrence and a lack of the occurrence of symptoms), forward reasoning, expertlike rules:

$$
\begin{aligned}
& \text { rule }_{1 \text { fevs }}: F=1 \wedge G=0 \wedge(F-G)=1 \longrightarrow m 1, \\
& \text { rule }_{2 \text { fevs }}: F=1 \wedge G=1 \wedge(F-G)=0 \longrightarrow m 2, \\
& \text { rule }_{3 \text { fevs }}: F=0 \wedge G=1 \wedge(F-G)=1 \longrightarrow m 3, \\
& \text { rule }_{4 \text { fevs }}: F=1 \wedge G=0 \wedge(F-G)=1 \longrightarrow a 1, \\
& \text { rule }_{5 \text { fevs }}: F=0 \wedge G=1 \wedge(F-G)=1 \longrightarrow a 2 .
\end{aligned}
$$

Observe that the preconditions of the rules 3 fevs and $5 f e v s$ as well as 1 fevs and 4 fevs are pairwise identical. However, the diagnosed faults are different. In fact, in the case of the singular fault assumption, the faults $m 3$ and $a 2$ are indistinguishable, and so are the faults $m 1$ and $a 1$.

For the analyzed case $(F=1, G=0$ and $(F-$ $G)=1$ ) only the rules 1 fevs and 4 fevs can be fired. The generated diagnoses are $m 1$ and $a 1$ (indistinguishable in the case of singular faults).

Note that assuming multiple faults, indistinguishability can be interpreted as a potential multiple fault. In the analyzed case the indicated diagnosis can be $D=$ $\{m 1, a 1\}$.

3.4. Case of forward, expert-like vertical weak diagnostic rules (fevw). The set of rules presented above in weak form is as follows:

$$
\begin{aligned}
& \text { rule }_{1 \text { fevw }}: F=1 \wedge(F-G)=1 \longrightarrow m 1, \\
& \text { rule }_{2 \text { fevw }}: F=1 \wedge G=1 \longrightarrow m 2, \\
& \text { rule }_{3 \text { fevw }}: G=1 \wedge(F-G)=1 \longrightarrow m 3, \\
& \text { rule }_{4 \text { fevw }}: F=1 \wedge(F-G)=1 \longrightarrow a 1, \\
& \text { rule }_{5 \text { fevw }}: G=1 \wedge(F-G)=1 \longrightarrow a 2 .
\end{aligned}
$$

Observe that the preconditions of the rules $3 f e v w$ and $5 f e v w$ as well as $1 f e v w$ and $4 f e v w$ are pairwise identical. However, the diagnosed faults are different. In fact, in the case of the singular faults assumption, the faults $m 3$ and $a 2$ are indistinguishable, and so are the faults $m 1$ and $a 1$.

For the analyzed case $(F=1, G=0$ and $(F-$ $G)=1$ ) only the rules 1 fevw and 4 fevw can be fired. The generated diagnoses are $m 1$ and $a 1$ (indistinguishable in the case of singular faults). 
Note that assuming multiple faults, indistinguishability can be interpreted as a potential multiple fault. In the analyzed case $(F=1, G=0$ and $(F-G)=1)$ the indicated diagnosis can be $D=\{m 1, a 1\}$.

\subsection{Case of forward, expert-like horizontal strong} diagnostic rules (feh). The set of rules generated with respect to horizontal interpretation of the matrix given by Table 3 is as follows:

$$
\begin{aligned}
& \text { rule }_{1 f_{e h}}: F=1 \longrightarrow m 1 \vee a 1 \vee m 2 \\
& \text { rule }_{2 f_{e h}}: G=1 \longrightarrow m 3 \vee a 2 \vee m 2 \\
& \text { rule }_{3 f_{e h}}:(F-G)=1 \longrightarrow m 1 \vee m 3 \vee a 1 \vee a 2
\end{aligned}
$$

In the examined case one can fire the first and the third rule. The potential diagnoses are $m 1, a 1, m 2, m 3$, and $a 2$ as well as, in fact, any combination of them in the case of admitting multiple faults. Note that both of the rules point to $m 1$ and $a 1$ so perhaps these diagnoses can be considered more likely than the other ones.

\subsection{Case of backward search with causal vertical} strong rules (bcvs). In the second basic way of interpretation following the direction from faults to signals (equivalent to causal inference), the generated set of rules is as follows:

$$
\begin{aligned}
& \text { rule }_{1 b c v s}: m 1 \longrightarrow F=1 \wedge G=0 \wedge(F-G)=1, \\
& \text { rule }_{2 b c v s}: m 2 \longrightarrow F=1 \wedge G=1 \wedge(F-G)=0, \\
& \text { rule }_{3 b c v s}: m 3 \longrightarrow F=0 \wedge G=1 \wedge(F-G)=1, \\
& \text { rule }_{4 b c v s}: a 1 \longrightarrow F=1 \wedge G=0 \wedge(F-G)=1, \\
& \text { rule }_{5 b c v s}: a 2 \longrightarrow F=0 \wedge G=1 \wedge(F-G)=1 .
\end{aligned}
$$

Note that conclusions of the rules $1 b c v s$ and $4 b c v s$ as well as $3 b c v s$ and $5 b c v s$ are pairwise identical. When applying these rules for the backward search of diagnoses in the analyzed case, we obtain two single fault diagnoses, namely, $m 1$ and $a 1$, which account for the observed symptoms. The interpretation (assuming minimal diagnoses) is that $m 1$ or $a 1$ must be faulty. It can be also the case that both of the elements are faulty, i.e., we have a multiple fault diagnosis $\{m 1, a 1\}$. However, in the context of the set of rules (5) such a diagnosis would not be a minimal one.

3.7. Case of backward search with causal vertical weak rules (bcvw). Applying the weak interpretation from faults to signals (equivalent to causal inference), the generated set of rules is as follows:

$$
\begin{aligned}
& \text { rule }_{1 b c v w}: m 1 \longrightarrow F=1 \wedge(F-G)=1, \\
& \text { rule }_{2 b c v w}: m 2 \longrightarrow F=1 \wedge G \\
& \text { rule }_{3 b c v w}: m 3 \longrightarrow G=1 \wedge(F-G)=1, \\
& \text { rule }_{4 b c v w}: a 1 \longrightarrow F=1 \wedge(F-G)=1, \\
& \text { rule }_{5 b c v w}: a 2 \longrightarrow G=1 \wedge(F-G)=1 .
\end{aligned}
$$

Note that conclusions of the rules $1 b c v w$ and $4 b c v w$ as well as $3 b c v w$ and $5 b c v w$ are pairwise identical. When applying these rules for backward search of diagnoses in the analyzed case, we obtain two single fault diagnoses, $m 1$ and $a 1$. The interpretation (assuming minimal diagnoses) is that $m 1$ or $a 1$ must be faulty. As above, it can be also the case that both of the elements are faulty, i.e., we have a multiple fault diagnosis $\{m 1, a 1\}$; however, in the context of the set of rules (5) such a diagnosis would not be a minimal one.

3.8. Case of backward search with horizontal causal rules (bch). Applying the horizontal interpretation from faults to signals (equivalent to causal inference), the generated set of rules is as follows:

$$
\begin{aligned}
& \text { rule }_{1 b c h}: m 1 \vee a 1 \vee m 2 \longrightarrow F=1, \\
& \text { rule }_{2 b c h}: m 3 \vee a 2 \vee m 2 \longrightarrow G=1, \\
& \text { rule }_{3 b c h}: m 1 \vee m 3 \vee a 1 \vee a 2 \longrightarrow(F-G)=1 .
\end{aligned}
$$

In the case considered the diagnoses are generated with the first and the third rule interpreted backwards; the potential single fault diagnoses are $m 1, a 1$ or $m 2$ in the case of the first rule and $m 1, m 3, a 1$ or $a 2$ in the case of the third rule. Further, since assuming the diagnoses $m 3, a 2$ and $m 2$ allows us to fire the rule $2 b c h$, and taking into account that $G=1$ is the conclusion of the second rule, these singular diagnoses might be eliminated, since they are inconsistent with the observed manifestations $(G=0)$. Hence the only singular diagnoses are $m 1$ and $a 1$. Note however, that this line of reasoning applies only under the assumption of a singular fault (no compensation phenomenon takes place).

Now, let us relax the assumption that a singular fault occurred. Hence a compensation can take place. Note that in order to fully explain the observed misbehavior $(F=1$ and $(F-G)=1)$, one has to employ both of the rules $1 b c h$ and $3 b c h$ at the same time. This is a crucial observation. In fact, a conjunction of symptoms can be explained with a set of two independent rules.

Now the problem is to find faults allowing firing both of the rules at the same time. There are two such singular faults, i.e., $m 1$ and $a 1$, and thus we have two singular diagnoses. Note, however, that in this specific case we can find two further, multiple-fault minimal diagnoses allowing us to fire both of the rules: these are $\{m 2, m 3\}$ and $\{m 2, a 2\}$. These multiple-fault diagnoses also fully explain the observed misbehavior assuming that the fault of $m 3$ compensates the influence of faulty $m 2$ at output $G$ and, similarly, that fault of $a 2$ compensates the fault of $m 2$ at output $G$.

3.9. Summary. The presented results are summarized in Table 4 The generated diagnoses are in most of the cases similar. The weakest model seems to be the one 
Table 4. Diagnostic results. Non-minimal diagnoses are marked with an asterisk.

\begin{tabular}{|c||c|c|}
\hline Rules & Singular faults & Multiple faults \\
\hline \hline fevs & $m 1, a 1$ & $\{m 1, a 1\}$ \\
\hline fevw & $m 1, a 1$ & $\{m 1, a 1\}$ \\
\hline feh & $m 1, a 1, a 2$ & $2^{\{m 1, a 1, a 2\}}$ \\
\hline bcvs & $m 1, a 1$ & $\{m 1, a 1\}^{*}$ \\
\hline bcvw & $m 1, a 1$ & $\{m 1, a 1\}^{*}$ \\
\hline bch & $m 1, a 1$ & $\{m 2, m 3\},\{m 2, a 2\}$ \\
\hline
\end{tabular}

based on the forward, expert-like horizontal interpretation of the diagnostic matrix - apart from the correct minimal single-fault diagnoses $m 1$ and $a 1$, it produces a diagnosis $a 2$ which, in the case of singular diagnoses, is obviously inconsistent with the observations. Due to the indistinguishability of faults, in the case of forward inference such faults can be interpreted as potential multiple faults by simply combining them into one set.

In the case of the backward search based on causal models, the generated diagnoses are the same in all three cases under the assumption of singular faults and provided that the hypothesized diagnoses are consistent with all manifestations. Multiple diagnoses generated without the assumption of minimal diagnoses are the same as in the case of forward, expert like rules.

The most interesting is the case of backward horizontal causal rules. By applying a more complex analysis in this case also the multiple-fault minimal diagnoses can be generated.

The core problem with the first five simple models is that none of them is capable of inferring the whole set of potential diagnoses, which is $m 1, a 1,\{m 2, a 2\}$, $\{m 2, m 3\}$ (Reiter, 1987). Only the last model allowed the correct results under a bit more complex analysis of a conjunction of rules explaining all the observed manifestations. Hence, perhaps a more advanced model should be incorporated.

The main problem with Table 3 is that in fact it corresponds to simple OR type causal graphs (Reggia et al., 1983; Reggia et al., 1985; Ligęza and FusterParra, 1997; Ligęza, 2004). For example, Table 3 corresponds to the causal graph presented in Fig. 2. The lowerlevel nodes $\{m 1, m 2, m 3, a 1, a 2\}$ are the initial causes, and the upper-level nodes $\{F, G, F-G\}$ are the observable manifestations. Using the classical FDI approach based on fault signatures (Kościelny, 2001), we obtain the results presented in Table 5. They are consistent with the ones which can be generated using the OR graph of Fig. 2 (assuming strong causality, i.e., if a fault occurs, the manifestation linked to it by an arrow must occur as well). Note that patterns such as $(1,0,0),(0,1,0),(0,0,1)$ are inconsistent. There are no faults influencing only one manifestation. Further, no multiple faults are detected with

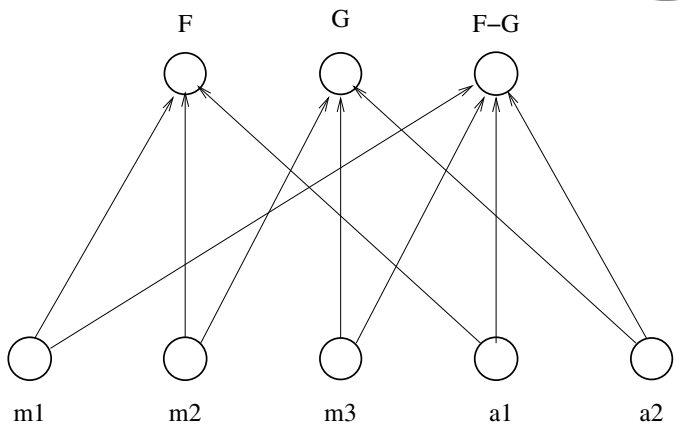

Fig. 2. OR causal graph for the multiplier-adder system.

Table 5. Generated diagnoses.

\begin{tabular}{|c||c|}
\hline $\begin{array}{c}\text { Signature for } \\
\text { (F,G,F-G) }\end{array}$ & $\begin{array}{c}\text { Singular } \\
\text { diagnoses }\end{array}$ \\
\hline \hline$(1,1,0)$ & $\{m 2\}$ \\
\hline$(1,0,1)$ & $\{m 1\},\{a 1\}$ \\
\hline$(0,1,1)$ & $\{m 3\},\{a 2\}$ \\
\hline$(1,1,1)$ & no singular fault \\
\hline
\end{tabular}

Table 3 although one can consider certain supersets of the singular faults as potential multiple fault diagnoses, provided that they are consistent with signal propagation rules for the system.

\section{Principles of multiple fault diagnosis}

The proposed approach follows the ideas of model-based consistency-based diagnostic inference founded on the analysis of the so-called conflict-sets and finding diagnoses as minimal hitting sets of them (Reiter, 1987) combined with causal graphs, as presented in (FusterParra, 1996; Ligęza and Fuster-Parra, 1997). The proposed ideas were first stated in (Ligęza, 2004) and recently developed in (Ligęza and Kościelny, 2007c).

The main conclusion of the presented analysis of the single-level diagnostic matrix approach and its rule-based interpretations is that the discussed approach is in practice limited to single-fault diagnosis. Only one type of rules, namely, the horizontal, causal ones, allowed a complete set of diagnoses. Further, it is often impossible to infer a unique diagnosis under the limited information available and the possibility of several causes having the same effect observed at the level of symptoms. The following underlying assumptions seem to constitute the basis for the understanding and organization of the diagnostic process in realistic, more complex cases:

- Diagnostics is more a process, and not a single action. Hence, it cannot be achieved by single-step forward inference based on precompiled knowledge. It requires more complex reasoning and auxiliary information gathering. 
- The diagnosis of complex systems may be based on the analysis of causal relationship and models of the system instead of expert-like knowledge; it can be also supported by expert and statistical knowledge.

- Causal inference is performed by backward search for hypotheses explaining the manifestations observed in the case of a failure.

- Two types of causal dependencies should be taken into account during the analysis, i.e., the AND combination of all the rules forming the observed pattern of symptoms, and the $O R$ combination of faults causing the occurrence of a certain symptom.

- Since all the necessary information is not available at the start of the diagnostic procedure, an incremental, knowledge-driven information gathering process is an intrinsic part of diagnostic inference.

- The direction of deviations constitutes useful qualitative information applicable to the refinement of diagnoses. Further, heuristic information, statistical and expert knowledge can be used to form preferences among diagnoses and ordering diagnostic tests.

The practical development of these ideas is presented below.

\section{Conjunctive and disjunctive faults}

Taking into account the conclusions following from the presented analysis, the concepts of conjunctive and disjunctive faults are introduced. They are necessary to express two types of causal rules used in the proposed approach.

Definition 1. A Conjunctive Conceptual Fault (a CCF, for short) is the hypothesis that several faults occur at the same time. A particular $C C F_{i}$ can be expressed as a set of faults, $C C F_{i}=\left\{f^{1}, f^{2}, \ldots, f^{j_{i}}\right\}$, or, logically, as a conjunction $C C F_{i}=f^{1} \wedge f^{2} \wedge \ldots \wedge f^{j_{i}}$.

In case all the faults belonging to a certain $C C F$ are confirmed and no more faults are observed, the $C C F$ becomes a diagnosis. Otherwise, it can be considered to be just a hypothetical or conceptual fault. If it fully explains the misbehavior of the system, it becomes a potential diagnosis for the system.

We shall also need the following definition of a Disjunctive Conceptual Fault (a $D C F$, for short) or an Intermediate Conceptual Fault (ICF). It occurs when a hypothesis is stated that at least one of a set of faults occurs.

Definition 2. A disjunctive conceptual fault or intermediate conceptual fault is the hypothesis that a certain set of components must contain a faulty component under some set of manifestations observed. A particular $D C F_{i}$ can be expressed as a set of faults, $D C F_{i}=\left\{f^{1}, f^{2}, \ldots, f^{j_{i}}\right\}$, or, logically, as a disjunction $D C F_{i}=f^{1} \vee f^{2} \vee \ldots \vee f^{j_{i}}$.

In other words, a $D C F$ (an $I C F$ ) is an imprecise localization of a fault with the accuracy of a set of system components. The indicated set of components is called a conflict set (Reiter, 1987) or an R-conflict (Cordier et al., 2000b; Cordier et al., 2000a). Here the idea of the DCF seems to be a useful notion for explaining the diagnostic approach in terms of the two-level approach.

5.1. Causal OR rules. The causal horizontal interpretations of the matrix with OR type rules is introduced below.

Definition 3. Let $g_{i}^{1}, g_{i}^{2}, \ldots, g_{i}^{j_{i}}$ be all the elements of the $i$-th row of matrix (1) taking the value of 1 and let $f^{1}, f^{2}, \ldots, f^{j_{i}}$ be the corresponding faults. A binary diagnostic table of the OR type is a table given by (1) with a set of OR rules of the the following form:

$$
\text { rule }_{i_{-} o r}: f^{1} \vee f^{2} \vee \ldots \vee f^{j_{i}} \longrightarrow m_{i}
$$

for $i=1,2, \ldots, J$.

This kind of OR matrix is close to the classical binary diagnostic matrices as discussed in (Kościelny, 2001; Kościelny, 2004b; Kościelny, 2004a). The main difference is that the rules read from the matrix are causal ones and not expert-like-the logical interpretation is from faults to manifestations.

The causal OR rules describe the fact that a single fault $f \in \mathbf{F}$ can cause the occurrence of some manifestations and a single manifestation can have numerous independent causes. This seems to be the most typical case in realistic systems. The intuition behind this model is that the correct operation of such systems depends on the correct work of numerous components, and if a single component fails, so does the overall system. Systems based on serially connected components are a perfect example here.

5.2. Causal AND rules. In some situations the causal model of the relationship between faults and manifestations can be of the AND type. In such a case only a conjunctive occurrence of several faults can evoke a manifestation.

Definition 4. Let $g_{i}^{1}, g_{i}^{2}, \ldots, g_{i}^{j_{i}}$ be all the elements of the $i$-th row of matrix (1) taking the value of 1 and let $f^{1}, f^{2}, \ldots, f^{j_{i}}$ be the corresponding faults. A binary diagnostic table of the AND type is a table given by (1) with a set of AND rules of the the following form:

$$
\text { rule }_{i_{-} a n d}: f^{1} \wedge f^{2} \wedge \ldots \wedge f^{j_{i}} \longrightarrow m_{i}
$$

for $i=1,2, \ldots, J$. 
This kind of AND rules and AND matrix is new with respect to the classical binary diagnostic matrices as discussed in (Kościelny, 2001; Kościelny, 2004b; Kościelny, 2004a). The main difference is that the rules read from the matrix are causal ones (i.e., as above) and the conjunction of faults may only evoke a manifestation.

\section{Adder: The two-level approach}

Consider once again the multiplier-adder system as presented in Fig. 1. Assume that, as before, $F=10$ and $G=12$, i.e., an incorrect output is observed at $F$. Recall that we shall refer mostly to the classical diagnostic problem defined as follows: The current state of the system is that the inputs are $A=3, B=2, C=2, D=3$ and $E=3$. It is easy to check that (if the system works correctly) the outputs should be $F=12$ and $G=12$. Since the current value of $F$ is incorrect, namely, $F=10$, the system is faulty. At least one of its components must be faulty. A further analysis of the case is presented in Section 3 .

Note that the value of $F$ is influenced by the inputs (observed) and the work of elements $m 1, m 2$ and $a 1$. If all the three elements work correctly, then the output would be correct. Since it is not, we can conclude that $D C F_{1}$ is observed: at least one of the elements $\{m 1, m 2, a 1\}$ must be faulty. Hence, a rule of the form

$$
\text { rule }_{1 \_o r}: m 1 \vee m 2 \vee a 1 \longrightarrow D C F_{1}
$$

can be stated.

A further analysis leads to the detection of $D C F_{2}$ : under the assumed manifestations, one of the elements $\{m 1, a 1, a 2, m 3\}$ must also be faulty. This is so since if all of them were correct, then $Z=C \cdot E$ calculated by $m 3$ must be equal to 6 , and since $G$ is observed to be $12, Y$ (calculated backwards and under the assumption that $a 2$ works correct) must also equal 6 . Hence, if $m 1$ is correct, then $X$ must be 6 as well, and if $a 1$ is correct, $F$ would be equal to 12. Since this is not the case, at least one of the components used must be faulty. So we have the following rule:

$$
\text { rule }_{2 \_o r}: m 1 \vee m 3 \vee a 1 \vee a 2 \longrightarrow D C F_{2} .
$$

Note that if $F$ is correct and $G$ is faulty, e.g., $F=12$ and $G=10$, then another $D C F$ observed would be $D C F_{3}$ equivalent to $\{m 2, m 3, a 2\}$ and so we have the third OR rule:

$$
\text { rule }_{3 \_o r}: m 2 \vee m 3 \vee a 2 \longrightarrow D C F_{3} .
$$

Moreover, $D C F_{2}$ equivalent to a fault in $\{m 1, m 3, a 1, a 2\}$ would occur as well.

If both of the outputs are incorrect (e.g., $F=10$ and $G=14$ ), then, in a general case, one can observe $D C F_{1}, D C F_{2}$ and $D C F_{3}$. Note, however, that whether
$D C F_{2}$ is a valid fault may depend on the observed outputs. For example, if $F=10$ and $G=10$ (both outputs incorrect but equal), then the structure and equations describing the work of the system do not lead to a conceptual fault (Cordier et al., 2000a; Cordier et al., 2000b).

Depending on the current manifestations, a $D C F$ can be observed (active) or not (inactive). For an effective diagnosis one needs only a specification of active $D C F$ s.

The diagnoses are calculated as reduced elements of the Cartesian product of the conflict sets associated to the active $D C F$ s. The reduction consists in the elimination of duplicates.

The OR matrix for the diagnosed system is presented in Table 6. The AND matrix defining the relationship be-

Table 6. OR binary diagnostic matrix for the adder system (the lower level).

\begin{tabular}{|c||c|c|c|c|c|}
\hline$D C F$ & $m 1$ & $m 2$ & $m 3$ & $a 1$ & $a 2$ \\
\hline \hline$D C F_{1}$ & 1 & 1 & & 1 & \\
\hline$D C F_{2}$ & 1 & & 1 & 1 & 1 \\
\hline$D C F_{3}$ & & 1 & 1 & & 1 \\
\hline
\end{tabular}

tween the $D C F$ s (active in the case of $F$ being incorrect and and $G$ correct) and the manifestations are presented in Table 7 Here $F^{*}, G^{*}$, etc. mean that the output is incor-

Table 7. AND binary diagnostic matrix for the adder system (the upper level).

\begin{tabular}{|c||c|c|c|}
\hline$M$ & $D C F_{1}$ & $D C F_{2}$ & $D C F_{3}$ \\
\hline \hline$F^{*}, G,(F-G)^{*}$ & 1 & 1 & \\
\hline$F, G^{*},(F-G)^{*}$ & & 1 & 1 \\
\hline$F^{*}, G^{*}, F-G$ & 1 & & 1 \\
\hline$F^{*}, G^{*},(F-G)^{*}$ & 1 & 1 & 1 \\
\hline
\end{tabular}

rect, while $F, G$, etc. denote the correct outputs observed at the corresponding variable.

In the analyzed case, i.e., $F$ being faulty and $G$ correct, the final diagnoses for the case considered are calculated as reduced elements of the Cartesian product of $D C F_{1}=\{m 1, m 2, a 1\}$ and $D C F_{2}=\{m 1, m 3, a 1, a 2\}$. There are the following potential diagnoses: $D_{1}=\{m 1\}$, $D_{2}=\{a 1\}, D_{3}=\{a 2, m 2\}$ and $D_{4}=\{m 2, m 3\}$. The final diagnoses in a general case are presented in Table 8

The calculation of diagnoses can be easily interpreted using AND/OR causal graphs (Ligęza and FusterParra, 1997; Ligęza, 2004). An appropriate AND/OR graph is presented in Fig. 3. The active links are represented with continuous lines while the potential ones with dashed lines. Active $D C F$ s are marked with thick-line open circles and the current diagnostic problem (manifestations) are also represented with a thick-line circle. The final diagnoses are calculated as the minimal sets of the 
Table 8. Final possible diagnoses.

\begin{tabular}{|c|c|}
\hline Manifestations & Diagnoses \\
\hline \hline$F^{*}, G,(F-G)^{*}$ & $\{a 1\},\{m 1\},\{a 2, m 2\},\{m 2, m 3\}$ \\
\hline$F, G^{*},(F-G)^{*}$ & $\{a 2\},\{m 3\},\{a 3, m 2\},\{m 1, m 2\}$, \\
\hline$F^{*}, G^{*},(F-G)$ & $\{m 2\},\{a 1, a 2\},\{a 1, m 3\}$, \\
& $\{a 2, m 1\},\{m 1, m 3\}$ \\
\hline$F^{*}, G^{*},(F-G)^{*}$ & $\{a 1, a 2\},\{a 1, m 2\},\{a 1, m 3\}$, \\
& $\{a 2, m 1\},\{a 2, m 2\},\{m 1, m 2\}$, \\
& $\{m 2, m 3\},\{m 1, m 3\}$ \\
\hline
\end{tabular}

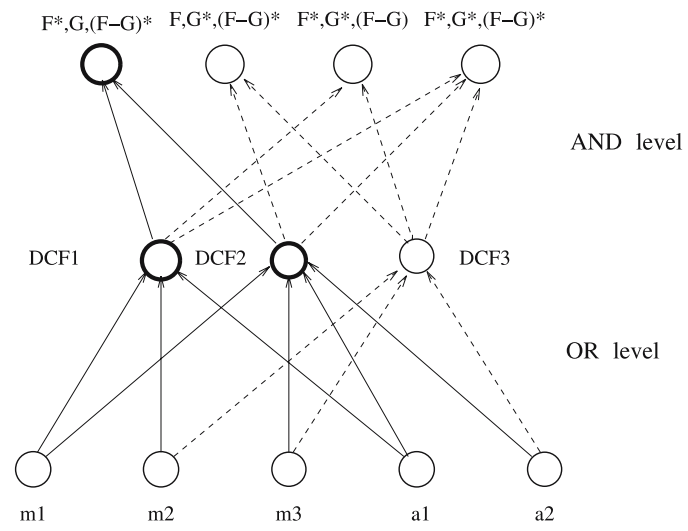

Fig. 3. AND/OR causal graph for the example multiplier-adder system.

lowest level elements which are necessary to satisfy the currently observed set of manifestations. The intermediate nodes representing the $D C F$ s are OR nodes while the top level nodes representing current manifestations are AND nodes.

The presented graphical interpretation can help us to formulate an algorithm for the diagnostic procedure.

\section{General diagnostic algorithm for $\mathrm{OR}$ and AND matrices and the two-level approach}

Consider a system to be diagnosed described by an OR matrix of Table 6 with $I$ manifestations of $D C F$ s (given by rows) and $K$ potential faults (specified with columns). Each fault corresponds to a certain system component. Further, let the second level be described by an AND matrix of the same generic shape, but with maximally $2^{J}$ combinations of manifestations (assigned to rows) and $I$ $D C F$ s (specified by columns).

Note that it is possible to combine these two matrices into a single one. In fact, by rotating the OR matrix by $90^{\circ}$ counterclockwise and concatenating it to the top row of the AND matrix, a composite matrix of $K+2^{J}$ rows and $I$ columns may be obtained. The algorithm to determine the set of all potential (and minimal) diagnoses is as follows:

1. Let $M^{+} \subset M$ be the set of manifestations to be diag- nosed. This set identifies a specific row of the AND matrix. All other rows, referring to other combinations of manifestations, need not be taken into examination. The specific row will be referred to as the active one. In Fig. 3 the active row corresponds to $\left.M^{+}=\left\{F^{*}, G,(F-G)^{*}\right\}\right)$ and it is represented by the top leftmost node marked with a thick-line open circle.

2. Determine the set of all $D C F$ s necessary to imply the combination of manifestations described with the active row. Let this be $D C F^{1}, D C F^{2}, \ldots, D C F^{i}$. In the example under consideration (manifestations given by $\left.F^{*}, G,(F-G)^{*}\right)$, these are $D C F_{1}$ and $\mathrm{DCF}_{2}$.

3. Determine the rows of the matrix OR defining $D C F^{1}, D C F^{2}, \ldots, D C F^{i}$. Let these be rows $j^{1}, j^{2}, \ldots, j^{i}$. In the example under consideration (manifestations given by $F^{*}, G,(F-G)^{*}$ ), these are rows 1 and 2 .

4. For any row $j \in\left\{j^{1}, j^{2}, \ldots, j^{i}\right\}$ read the contents of appropriate $D C F_{j}=\left\{f_{j}^{1}, f_{j}^{2}, \ldots, f_{j}^{k}\right\}$. In the example under consideration (manifestations given by $\left.F^{*}, G,(F-G)^{*}\right)$, we have just two $D C F$ s, i.e., $D C F_{1}=\{m 1, m 2, a 1\}$ and $D C F_{2}=$ $\{m 1, m 3, a 1, a 2\}$.

5. Find the set $\mathbf{D}=\left\{D_{1}, D_{2}, \ldots, D_{d}\right\}$ of all minimal diagnoses, where each $D \in \mathbf{D}$ is a minimal hitting set for all the sets $D C F^{1}, D C F^{2}, \ldots, D C F^{i}$, i.e., $D \cap D C F^{j} \neq \emptyset$ for any $j \in\{1,2, \ldots, i\}$ and $D$ is minimal.

6. The potential diagnoses are given by $D_{1}, D_{2}, \ldots, D_{d}$. Here we have four minimal diagnoses, i.e., $D_{1}=\{m 1\}, D_{2}=\{a 1\}$, $D_{3}=\{a 2, m 2\}$ and $D_{4}=\{m 2, m 3\}$.

Note that $\mathbf{D}$ can be found by calculating the Cartesian product $D C F^{1} \times D C F^{2} \times \cdots \times D C F^{i}$ and further reducing all its elements by eliminating repetitions of elements. It can also be found by the backward search of the AND/OR graphs for all minimal sets of elementary faults logically entailing the current observations (Ligęza and Fuster-Parra, 1997; Ligęza, 2004).

\section{Improving the efficiency of diagnosis}

8.1. Qualitative evaluation of faults. A most popular classification of faults is the binary one. An element can be just faulty $(f=1)$ or not $(f=0)$. This kind of classification is prevailing in technological systems, sometimes extended to several degrees or a fuzzy fault description.

Note, however, that in some particular cases the fault can be interpreted as a significant deviation from some 
expected status or value, and the deviation has not only an amplitude, but a direction or a sign as well. In this case the fault can be said to be negative or positive, and a classification described with three values $\{-, 0,+\}$ can be established. This kind of knowledge can be used for a further refinement of diagnoses without taking additional measurements, tests or observations. Further, the same classification can be assigned also to manifestations, i.e., values of certain variables can be normal (0), below the norm $(-)$ or above it $(+)$.

8.2. Elimination of spurious diagnoses. The idea is that in many cases the influence of faults on the manifestations can be analyzed in a qualitative way using the three-valued approach. Two key observations may be useful: (i) some faults can be only negative or only positive, and (ii) the defined sign of the deviation of a fault also defines the sign of the deviation of the influenced manifestation. For example, the voltage of a battery can only be normal $(0$, no fault) or low $(-$, below normal). The level of liquid in a tank can be normal (0), low (-), or high $(+)$. The clock can be exact, but when faulty, it can slow down $(-)$ or advance $(+)$.

The influence of a fault on a manifestation can be marked using the sign. For example, low battery (battery_fault) causes low light (light_fault), i.e., battery_fault $(-) \longrightarrow$ light_fault $(-)$. Assuming that both the multipliers and adders of the diagnosed system when faulty produce results lower than expected, we can specify the following influences:

$$
\begin{aligned}
& m 1 \longrightarrow F(-), \\
& m 2 \longrightarrow F(-), \\
& m 2 \longrightarrow G(-), \\
& m 3 \longrightarrow G(-), \\
& a 1 \longrightarrow F(-), \\
& a 2 \longrightarrow G(-),
\end{aligned}
$$

Obviously, the diagnosis must be consistent with the set of rules defining qualitative influences. Diagnosis $D_{1}=\{m 1\}$ is consistent with 13 : faulty $m 1$ causes a lower value of $F$. The same applies to $D_{2}=\{a 1\}$. Note, however, that in the case of $D_{3}=\{m 2, a 2\}$ also the value of $G$ should be lower. Since it is not, the diagnosis is inconsistent and it can be eliminated. The same applies to $D_{4}=\{m 2, m 3\}$.

In a general case, the influence of the components of the multiplier-adder system can be described with the following set of rules:

$$
\begin{array}{ll}
m 1(-) \longrightarrow F(-), & m 1(+) \longrightarrow F(+), \\
m 2(-) \longrightarrow F(-), & m 2(+) \longrightarrow F(+), \\
m 2(-) \longrightarrow G(-), & m 2(+) \longrightarrow G(+), \\
m 3(-) \longrightarrow G(-), & m 3(+) \longrightarrow G(+), \\
a 1(-) \longrightarrow F(-), & a 1(+) \longrightarrow F(+), \\
a 2(-) \longrightarrow G(-), & a 2(+) \longrightarrow G(+) .
\end{array}
$$

In this case none of the four diagnoses can be eliminated. However, more detailed characteristics of the diagnoses can be found. Let us introduce a definition of qualitative diagnosis taking into account the deviation sign of a fault.

Definition 5. A qualitative diagnosis

$$
D=\left\{d_{1}(\#), d_{2}(\#), \ldots, d_{k}(\#)\right\}
$$

is a diagnosis fully explaining the observed misbehavior and covering the knowledge of the deviation sign for any fault (if accessible). Here \# is + if the deviation sign is positive, - if the deviation sign is negative and ? if the deviation sign is unknown (any, undetermined).

In the case of the analyzed failure, we have the following qualitative diagnoses consistent with the observations:

- $D_{1}=\{m 1(-)\}$; if the fault concerns $m 1$ only, then $m 1$ produces a lower output,

- $D_{2}=\{a 1(-)\}$; if the fault concerns $a 1$ only, then $a 1$ produces a lower output,

- $D_{3}=\{m 2(-), a 2(+)\}$ if the fault concerns $m 2$ and $a 2$, then $m 2$ produces a lower output, while $a 2$ produces a higher output,

- $D_{4}=\{m 2(-), m 3(+)\}$ if the fault concerns $m 2$ and $m 3$, then $m 2$ produces a lower output, while $m 3$ produces a higher output.

A further technical analysis can be useful. For example, some deviations are highly improbable. Hence, a preference relation among diagnoses can be established.

8.3. Elimination algorithm. Assume that we have a set $R=\left\{r_{1}, r_{2}, \ldots, r_{r}\right\}$ of qualitative influence rules of the form (14). Further, let a set of qualitative observations concerning system variables of the form $V=$ $\left\{V_{1}, V_{2}, \ldots, V_{v}\right\}$ be given where each of the variables is described as $V_{i}(-), V_{i}$, or $V_{i}(+)$ (i.e., lower than expected, normal, or higher than the expected value). Let $D \in \mathbf{D}$ be a certain diagnosis, $D=\left\{c_{1}, c_{2}, \ldots, c_{c}\right\}$. Any such diagnosis can produce as many as $2^{c}$ qualitative diagnoses $D Q=\left\{d_{1}, d_{2}, \ldots, d_{c}\right\}$, where each $d_{j} \in D Q$ is either $d_{j}=c_{j}(-)$ or $d_{j}=c_{j}(+)$.

The outline of an algorithm for finding qualitative diagnoses and eliminating spurious ones is as follows:

1. For any diagnosis $D$ find all possible qualitative diagnoses $D Q$. Then examine each qualitative diagnosis $D Q$ in turn.

2. For any qualitative diagnosis $D Q$ fire all the applicable rules of $R$ and find all the qualitative variables resulting from this operation. Note that if there are two 
or more fired rules concerning the same variable, the obtained set may be inconsistent (e.g., certain variable should simultaneously increase and decrease).

3. Reduce the obtained set of qualitative variables to a consistent set. Apply the qualitative composition of Table 9. Repetitions of facts are thus eliminated.

Table 9. Qualitative composition of variables.

\begin{tabular}{|c||c|c|c|}
\hline & - & 0 & + \\
\hline \hline- & - & - & $?$ \\
\hline 0 & - & 0 & + \\
\hline+ & $?$ & + & + \\
\hline
\end{tabular}

4. Check if the obtained reduced set of qualitative variables is consistent with the current observations. It is inconsistent if there exists a variable having a different qualitative deviation assigned in these sets. In this case, eliminate the qualitative diagnosis $D Q$ as a spurious one.

As an example, consider the diagnosis $D_{3}=$ $\{m 2, a 2\}$. In this case we have to analyze the following potential qualitative diagnoses:

- $D Q_{3,1}=\{m 2(-), a 2(-)\}$,

- $D Q_{3,2}=\{m 2(-), a 2(+)\}$,

- $D Q_{3,3}=\{m 2(+), a 2(-)\}$,

- $D Q_{3,4}=\{m 2(+), a 2(+)\}$.

Let us analyze these diagnoses in turn.

In the case of $D Q_{3,1}=\{m 2(-), a 2(-)\}$, one can infer a qualitative facts set of the form $\{F(-), G(-), G(-)\}$. The reduced set of facts is $\{F(-), G(-)\}$. It is inconsistent with the observations which are $\{F(-), G(0)\}$.

In the case of $D Q_{3,2}=\{m 2(-), a 2(+)\}$, one can infer a qualitative facts set of the form $\{F(-), G(-), G(+)\}$. The reduced set of facts is $\{F(-), G(?)\}$. Since $G(?)$ is consistent with anything, this diagnosis is consistent.

In the case of $D Q_{3,3}=\{m 2(+), a 2(-)\}$, one can infer a qualitative facts set of the form $\{F(+), G(+), G(-)\}$. The reduced set of facts is $\{F(+), G(?)\}$. This set is inconsistent with the observations which are $\{F(-), G(0)\}$.

In the case of $D Q_{3,4}=\{m 2(+), a 2(+)\}$ one can infer a qualitative facts set of the form $\{F(+), G(+), G(+)\}$. The reduced set of facts is $\{F(+), G(+)\}$. This set is inconsistent with the observations which are $\{F(-), G(0)\}$.

To summarize, there is only one qualitative diagnosis. This is $D Q_{3,2}=\{m 2(-), a 2(+)\}$, and it is consistent with the observations.

\section{Concluding remarks}

Limitations of the direct application of diagnostic matrices in the case of multiple fault diagnosis have been presented and examined. The main problem with the binary decision matrix-based approach is that in realistic systems it is very seldom that the set of observed manifestations provides enough information to assure deterministic diagnosability, i.e., all the faults (even in the case of singular faults) have different signatures. In some most typical cases a single manifestation may have numerous faults as potential causes. Hence, a kind of hypothetical reasoning, based on abduction, seems rational in the case of more complex systems. The explanation for this is simple: using the classical OR graph causal model, the most typical situation is that there are several (or even numerous) potential faults in the lower level of initial causes, and only one (or few) manifestations observable in the upper level.

It is shown that the knowledge expressed with a binary diagnostic matrix, although interpreted in several ways, including expert-like, forward reasoning models and causal, backward reasoning ones, does not lead to the production of all potential diagnoses in the case of multiple faults. Hence the direct use of a single diagnostic matrix, especially the one interpreted in a forward, expertlike manner, is of limited use in more complex systems with hidden variables and interactions.

Simultaneously, it can be seen that the model defined with a diagnostic matrix should be extended to cover more complex inference possibilities. Multiple faults must be taken into account. Moreover, the information about sets of elements such that at least one of them is faulty (the so-called conflict sets (Reiter, 1987)) must be expressible.

Hence, a proposal of extending the expressive power of traditional binary diagnostic matrices covers both including causal relationship between faults and manifestations and logical connectives. The main idea of the proposal is three-fold and consists in:

- providing a new interpretation of the matrices with rules - the new rules should follow the causal direction of inference (i.e., from faults, which are the initial causes, to manifestations),

- introducing two types of diagnostic matrices, each of them having a different logical interpretation, one with logical OR type meaning and another one with logical AND type meaning,

- introducing a two-level knowledge representation with OR matrices at the lower level and AND matrices in the upper one,

As a result, a new diagnostic model, composed of two matrices forming two levels, is put forward. One of the matrices defines the so-called intermediate conceptual faults 
and is analogous to the classical OR matrix, while the second level matrix combines the influence of the conceptual faults onto manifestations and is a new type AND matrix. A diagnostic algorithm is presented.

The proposed approach combines in fact the consistency-based diagnosis of (Reiter, 1987) and the AND/OR graphs (Ligęza and Fuster-Parra, 1997) into a technical approach similar to FDI (Kościelny, 2001). Some characteristic features of the proposed approach are as follows:

- it is based on causal models, including AND and OR type of causal relations,

- it allows the diagnosis of multiple faults by applying a backward (abductive) search,

- a set of potential diagnoses is generated, including both single and multiple faults,

- a refinement procedure can be applied if qualitative information about the sign of deviations is available.

If auxiliary knowledge about the direction of deviations and their mutual influences is available, the set of obtained diagnoses can be further refined. Qualitative diagnoses indicating the type of fault (i.e., deviation up or down) are available. The model can also be extended towards using heuristic and expert-like knowledge, introducing more levels (hierarchic diagnosis) and constraints for elimination of diagnoses, and ordering test procedures. For some ideas, see (Ligęza and FusterParra, 1997; Ligęza, 2004).

\section{Acknowledgment}

The support from the Polish Ministry of Science and Higher Education under the grant ALDIAG no. $1527 / \mathrm{T} 11 / 2005 / 29$ is kindly acknowledged.

\section{References}

Cordier M.-O., Dague P., Dumas M., Lévy F., Montmain J., Staroswiecki M., Travé-Massuyès L. (2000a). AI and automatic control approaches of model-based diagnosis: Links and underlying hypotheses, Proceedings of the IFAC Symposium SAFEPROCESS, Budapest, Hungary, pp. 274-279.

Cordier M.-O., Dague P., Dumas M., Lévy F., Montmain J., Staroswiecki M., Travé-Massuyès L. (2000b). A comparative analysis of AI and control theory approaches to modelbased diagnosis, Proceedings of 14th European Conference on Artificial Intelligence, Berlin, Germany, pp. 136140.

Davis R. and Hamscher W. (1992). Model-based reasoning: Troubleshooting, in W. Hamscher, L. Console and J. de Kleer (Eds.), Readings in Model-Based Diagnosis, Morgan Kaufmann Publishers, San Mateo, CA, pp. 3-24.
Frank P. (1990). Fault diagnosis in dynamic systems using analitical and knowledge-based redundancy - A survey and some new results, Automatica 26(3): 459-474.

Frank P. (1996). Analytical and qualitative model-based fault diagnosis - A survey and some new results, European Journal of Control 2(1): 6-28.

Fuster-Parra P. (1996). A model for causal diagnostic reasoning. Extended inference modes and efficiency problems, Ph.D. thesis, University of Balearic Islands.

Hamscher W., Console L. and de Kleer J. (Eds.) (1992). Readings in Model-Based Diagnosis, Morgan Kaufmann, San Mateo, CA.

Korbicz J., Kościelny J., Kowalczuk Z. and Cholewa W. (Eds.) (2004). Fault Diagnosis. Models, Artificial Intelligence, Applications, Springer-Verlag, Berlin.

Kościelny J. M. (2001). Diagnosis of Automated Industrial Processes, Akademic Publishing House EXIT, Warsaw, (in Polish)

Kościelny J. M. (2004a). Methodology of process diagnosis, in J. Korbicz, J. Kościelny, Z. Kowalczuk and W. Cholewa Fault Diagnosis. Models, Artificial Intelligence, Applications, Springer-Verlag, Berlin, pp. 57-114.

Kościelny J. M. (2004b). Models in process diagnosis, in J. Korbicz, J. Kościelny, Z. Kowalczuk and W. Cholewa, Fault Diagnosis. Models, Artificial Intelligence, Applications, Springer-Verlag, Berlin, pp. 29-43.

Ligęza A. (2004). Selected methods of knowledge engineering in system diagnosis, in J. Korbicz, J. Kościelny, Z. Kowalczuk and W. Cholewa Fault Diagnosis. Models, Artificial Intelligence, Applications, Springer-Verlag, Berlin, pp. 633-668.

Ligęza A. and Fuster-Parra P. (1997). And/or/not causal graphs - A model for diagnostic reasoning, International Journal of Applied Mathematics and Computer Science 7(1): 185-203.

Ligęza A. and Kościelny J. M. (2007a). Diagnosis of single and multiple faults. A combination of graph-based and algebraic approach, in R. Tadeusiewicz, A. Ligęza and M. Szymkat (Eds.), Computer Methods and Systems, Vol. CMS'2007, AGH University of Science and Technology, ONT, Cracow, pp. 123-128.

Ligęza A. and Kościelny J. M. (2007b). Diagnosis of single and multiple faults. An analysis of algebraic approach based on diagnostic matrix and rule-based interpretations, in R. Tadeusiewicz, A. Ligęza and M. Szymkat (Eds.), Computer Methods and Systems, Vol. CMS'2007, AGH University of Science and Technology, ONT, Cracow, pp. 117-122.

Ligęza A. and Kościelny J. M. (2007c). A new approach to multiple fault diagnosis. Combination of diagnostic matrices, graphs, algebraic and rule-based models. The case of two-layer models, in J. Korbicz, K. Patan and M. Kowal (Eds.), Fault Diagnosis and Fault Tolerant Control, Academic Publishing House EXIT, Warsaw, pp. 219-230. 
Reggia J. A., Nau D. S. and Wang P. Y. (1983). Diagnostic expert system based on a set covering model, International Journal on Man-Machine Studies 19(5): 437-460.

Reggia J. A., Nau D. S. and Wang P. Y. (1985). A formal model of diagnostic inference. Problem formulation and decomposition, Information Sciences 37(1-3): 227-256.

Reiter R. (1987). A theory of diagnosis from first principles, Artificial Intelligence 32(1): 57-95.
Tzafestas S. (1989a). System fault diagnosis using the knowledge-based methodology, in Tzafestas S. (Ed.) Knowledge-Based System Diagnosis, Supervision and Control, Plenum Press, New York, NY/London, pp. 509595.

Tzafestas S. (Ed.) (1989b). Knowledge-Based System Diagnosis, Supervision and Control, Plenum Press, New York, NY/London.

Received: 3 November 2007

Revised: 14 May 2008 